\title{
NON-LINEAR VIBRATION OF TIMOSHENKO BEAMS BY FINITE ELEMENT METHOD
}

\author{
Jerzy Rakowski, Michą Guminiak \\ Poznań University of Technology, Institute of Structural Engineering, Poznań, Poland \\ e-mail: jerzy.rakowski@put.poznan.pl; michal.guminiak@put.poznan.pl
}

\begin{abstract}
The paper is concerned with free vibrations of geometrically non-linear elastic Timoshenko beams with immovable supports. The equations of motion are derived by applying the Hamilton principle. The approximate solutions are based on the negligence of longitudinal inertia forces but inclusion of longitudinal deformations. The Ritz method is used to determine non-linear modes and the associated non-linear natural frequencies depending on the vibration amplitude. The beam is discretized into linear elements with independent displacement fields. Consideration of the beams divided into the regular mesh enables one to express the equilibrium conditions for an arbitrary large number of elements in form of one difference equation. Owing to this, it is possible to obtain an analytical solution of the dynamic problem although it has been formulated by the finite element method. Some numerical results are given to show the effects of vibration amplitude, shear deformation, thickness ratio, rotary inertia, mass distribution and boundary conditions on the non-linear natural frequencies of discrete Timoshenko beams.
\end{abstract}

Keywords: Timoshenko beams, non-linear vibrations, eigenvalue problem, finite elements

\section{Introduction}

The linear free vibration of Timoshenko beams was studied by Levinson and Cooke (1982). Bhashyam and Prathap (1981) applied the Finite Element Method and elements with linear shape functions to determine natural frequencies of Timoshenko beams. The authors eliminated the shear locking by a selective reduced integrations procedure. The studies of beams with immovable supports weere carried out by Woinowsky-Krieger (1950), Hsu (1960), Evensen (1968) and Lewandowski (1987). The authors used a continuum approach as well as the finite element approach proposed by Levinson and Cooke (1982), Sarma and Varadan (1983) and Kitipornchai et al. (2009). The exact solutions given in form of elliptic functions were presented by Woinowsky-Krieger (1950) and Hsu (1960). Evensen (1968) analysed beams with various boundary conditions using the perturbation method. Lewandowski (1987) applied the Ritz method to obtain the frequency-amplitude relationship for elastically supported beams. The solutions presented by Bhashyam and Prathap (1980) as well as Sarma and Varadan (1983) are based on the assumption that the equation of motion is satisfied only at the instant of the maximum deformation. Dumir and Bhaskar (1988) compared the results of analysis of non-linear vibrations of beams and plates obtained by various methods. They also brought out the source of errors which appear in some finite elements formulations, e.g. presented by Mei and Decha-Umphai (1985). Marur and Prathap (2005) solved the non-linear vibration problem of Timoshenko beams by applying variational formulations, and similar isssues for thick asymetric beams were inastigated in a wide range by Singh and Rao (1998). The problem of non-linear vibration of Timoshenko beams taking into account cracking was invastigated by Kitipornchai et al. (2009). The non-linear formulation of the Timoshenko beam based on the modified couple stress theory was applied by Asghari et al. (2010) to static and free vibration analysis of beams. 
Effectiveness of the Finite Element Method depends e.g. on the type of finite elements taken in the analysis. Many of them induce undesirable parasitic effects as shear locking. A critical analysis of a class of finite elements was carried out by Rakowski (1990, 1991a, 1991b). In the present work the linear finite element developed by Rakowski (1990) is used in the analysis of non-linear vibration of the Timoshenko beam. Such an improved linear element does not lock and gives a better convergence to the exact results than the reduced-integrated one, and the division of considered beams into the regular mesh of identical elements enables one to formulate the equilibrium conditions in form of one difference equation. The advantage of this FEM formulation idea is that the solution of the considered dynamic problem can be obtained in an analytical closed form and that it facilitates analyses regardless of the number of finite elements. The solving procedure is analogous to that used for continuous systems, but in this case the nodal displacements are spatial functions of the discrete variable (the index number of a node). This methodology was adopted to solve the problem of vibrations of infinite Bernoulli-Euler beams by Rakowski and Wielentejczyk (1996, 2002). In the present approach, the equations of motion for a continuous beam are derived from the variational formulation by applying Hamilton's principle. The approximate time function is assumed to be harmonic and the solution of the problem is based on the idea presented by Rosenberg (1966) and Szemplińska-Stupnicka (1983) where non-linear normal modes and natural frequencies of vibrations are unknown and amplitude dependent. The effects of shear deformation, thickness ratio and rotary inertia on the non-linear natural frequencies are analysed. The element stiffness matrix and the consistent mass matrix are derived using the simple linear elements with independent displacement fields. The shear locking in exactly integrated elements is eliminated by introducing a scalling factor into the stiffness matrix (Rakowski, 1990). Beams with different boundary conditions are considered. The comparison of results obtained for the evaluated elements and the reduced-integrated ones is given.

\section{Theoretical considerations}

The equation for the strain energy for a beam of length $l$, including shear deformation, axial force, longitudal and the large transverse displacement effect, is

$$
\begin{aligned}
U= & \int_{0}^{l} \frac{E A}{2}\left(u_{, x}-\frac{1}{2} w_{, x}^{2}\right)^{2} d x+\int_{0}^{l} \frac{E J}{2} \theta_{, x}^{2} d x+\int_{0}^{l} \frac{\kappa G A}{2}\left(w_{, x}-\theta\right)^{2} d x \\
& +\int_{0}^{l} H\left(u_{, x}-\frac{1}{2} w_{, x}^{2}\right)^{2} d x
\end{aligned}
$$

where $u, w$ are the longitudinal and transverse displacements of the centroidal axis, $\theta$ is the rotation of the beam section, $E, G$ are the elastic and shear moduli, $J, A$ are the moment of inertia and the cross-section area, respectively, $H$ is the initial axis force and $\kappa$ is the shear coefficient. The kinetic energy including horizontal and rotary inertias can be expressed as

$$
T=\int_{0}^{l} \frac{m}{2} u_{, t}^{2} d x+\int_{0}^{l} \frac{m}{2} w_{, t}^{2} d x+\int_{0}^{l} \frac{m \rho^{2}}{2} \theta_{, t}^{2} d x
$$

where $m$ is mass per unit length and $\rho$ is the radius of gyration of the cross section.

Equations (2.1) and (2.2) give, on applying Hamilton's principle, the following equations of motion 


$$
\begin{aligned}
& E A\left[u_{, x}+\frac{1}{2}\left(w_{, x}\right)^{2}\right]_{, x}-m u_{, t t}=0 \\
& \left\{H w_{, x}+E A\left[u_{, x}+\frac{1}{2}\left(w_{, x}\right)^{2}\right] w_{, x}\right\}_{, x}+\kappa G A\left[w_{, x}-\theta\right]_{, x}-m_{, t t}=0 \\
& E J \theta_{, x x}+\kappa G A\left(w_{, x}-\theta\right)-m \rho^{2} \theta_{, t t}=0
\end{aligned}
$$

Neglecting the horizontal inertia forces in the second equation of (2.3), yields

$$
C=E A\left[u_{, x}+\frac{1}{2}\left(w_{, x}\right)^{2}\right]_{, x}=\mathrm{const}
$$

Integrating expression (2.4) in the range from 0 to $l$ for an immovable beam, we have

$$
C=\int_{0}^{l} \frac{E A}{2 a} w_{, x}^{2} d x
$$

Substituting (2.4) into equation (2.3) and eliminating the unknown function $\theta$, yields

$$
\begin{aligned}
& \left(\frac{H+C}{\kappa G A}+1\right) \frac{\partial^{4} w}{\partial x^{4}}-\frac{H+C}{E J} \frac{\partial^{2} w}{\partial x^{2}}-\left[\frac{m}{\kappa G A}+\frac{m \rho^{2}}{E J}\left(\frac{H+C}{\kappa G A}+1\right)\right] \frac{\partial^{4} w}{\partial x^{2} \partial t^{2}} \\
& \quad+\frac{m}{E J} \frac{\partial^{2} w}{\partial t^{2}}+\frac{m^{2} \rho^{2}}{\kappa E J G A} \frac{\partial^{4} w}{\partial t^{4}}=0
\end{aligned}
$$

Let us assume the approximate solution to equation (2.6) to be harmonic function of time (Woinowsky-Krieger, 1950)

$$
w(x, t)=\alpha W(x) \cos \omega t
$$

where $W(x)$ is a function of the spatial variable (not given a priori), $\alpha$ is the vibration amplitude of an arbitrary point $x_{0}$ of the beam axis for which $W\left(x_{0}\right)=1$. The non-linear normal mode $W(x)$ and the free frequency vibrations $\omega$ depend on the boundary conditions and on the vibration amplitude $\alpha$. The above solution does not satisfy non-linear equation (2.6) at every time. We minimize the error by equating the weighed residual of equation (2.6) to zero for one time period with the weighting function cos $\omega t$ (Dumir and Bhaskar, 1988; Szemplińska-Stupnicka, 1983). As a result, the equilibrium equation in the dimensionless form is obtained

$$
\frac{d^{4} W}{d \xi^{4}}+\frac{1}{F}\left[\Omega^{2} \frac{F+f}{\lambda^{2}}-(s+c) \lambda^{2}\right] \frac{d^{2} W}{d \xi^{2}}+\frac{\Omega^{2}}{F}\left(f \frac{\Omega^{2}}{\lambda^{4}}-1\right) W=0
$$

where

$$
\begin{array}{lll}
W=W(\xi) & \xi=\frac{x}{L} & f=\frac{2(1+\nu)}{\kappa} \\
s=\frac{H}{E A} & \lambda=\frac{L}{\rho} & \Omega=\frac{m \omega^{2} L^{4}}{E J} \\
c=\frac{3}{8} \frac{\delta^{2}}{\lambda^{2}} \int_{0}^{1} \frac{d W}{d \xi} d \xi & \delta=\frac{\alpha}{\rho} & F=\frac{s+c}{f}+1
\end{array}
$$

and $\nu$ is Poisson's ratio. The solution tf equation (2.8) is

$$
W(x)=C_{1} \sinh \mu_{1} \xi+C_{2} \cosh \mu_{1} \xi+C_{3} \sin \mu_{2} \xi+C_{4} \cos \mu_{2} \xi
$$

where $C_{i}$ are arbitrary constants and

$$
\begin{array}{ll}
\mu_{1}^{2}=\sqrt{\frac{b^{2}}{4}-\frac{\Omega}{F}\left(\frac{f \Omega^{2}}{\lambda^{4}}-1\right)}+\frac{b}{2} \quad \mu_{2}^{2}=\sqrt{\frac{b^{2}}{4}-\frac{\Omega}{F}\left(\frac{f \Omega^{2}}{\lambda^{4}}-1\right)}-\frac{b}{2} \\
b=\frac{1}{F}\left[\frac{\Omega^{2}}{\lambda^{2}}(F+f)-(s+c) \lambda^{2}\right]
\end{array}
$$


From the third equation of (2.3) one finds that the dimensionless rotation function depending on the spatial variable is of the form

$$
\phi(\xi)=\left(C_{1} \sinh \mu_{1} \xi+C_{2} \cosh \mu_{1} \xi\right) \frac{\beta_{1}}{\mu_{1}}-\left(C_{3} \cos \mu_{2} \xi+C_{4} \sin \mu_{2} \xi\right) \frac{\beta_{2}}{\mu_{2}}
$$

where $\beta_{1}=f \Omega^{2} / \lambda^{2}+F \mu_{1}^{2}$ and $\beta_{2}=f \Omega^{2} / \lambda^{2}-F \mu_{2}^{2}$.

The boundary conditions for beams with immovable supports are:

— for the hinged end

$$
W=0 \quad M=E J \frac{d \phi}{d \xi}=0
$$

— for the clamped end

$$
W=0 \quad \phi=0
$$

The frequency equations derived for several combinations of the boundary conditions are: — for the hinged-hinged beam

$$
\sinh \mu_{1} \sin \mu_{2}=0
$$

— for the clamped-clamped beam

$$
2+\frac{\beta_{2}^{2} \mu_{1}^{2}-\beta_{1}^{2} \mu_{2}^{2}}{\beta_{1} \beta_{2} \mu_{1} \mu_{2}} \sinh \mu_{1} \sin \mu_{2}-2 \cosh \mu_{1} \cos \mu_{2}=0
$$

— for the clamped-hinged beam

$$
\beta_{2} \mu_{1} \tanh \mu_{1}+\beta_{1} \mu_{2} \tanh \mu_{2}=0
$$

Since the non-linear frequency parameter $\Omega^{2}$ and the non-linear vibration mode $W$ depend on the vibration amplitude $\alpha$, the eigenvalue problem of non-linear equation (2.8) is solved iteratively (Lewandowski, 1987). For hinged-hinged beams, the non-linear mode is identical to the linear one and independent of the amplitude. Hence the frequency parameter $\Omega^{2}$ can be expressed in an analytical form as the root of the quadratic equation

$$
f \frac{\Omega^{4}}{\lambda^{4}}-\Omega^{2}\left[(F+k) \frac{k^{2} \pi^{2}}{\lambda^{2}}+1\right]+k^{2} \pi^{2}\left[F k^{2} \pi^{2}+\lambda(s+c)\right]=0
$$

where $c=(3 / 16)\left(k^{2} \pi^{2} \delta^{2} / \lambda^{2}\right), k$ is the number of the vibration mode. Ommiting the rotary inertia effect, yields

$$
\Omega^{2}=k^{2} \pi^{2} \frac{F k^{2} \pi^{2}+\lambda^{2}(s+c)}{1+f \frac{k^{2} \pi^{2}}{\lambda^{2}}}
$$

By assuming in (2.14) or (2.15) $f=0$, one obtains non-linear frequencies for the Bernoulli-Euler beam in which the axial force effect is taken into account. Table 1 presents the rotary inertia effect $I_{\rho}$ on the non-linear frequency ratio $\omega / \omega_{1}$ for the fundamental vibration mode of simply-supported beams ( $\alpha$ is the amplitude in the middle of the beam, $\Omega_{1}$ is the frequency parameter determined for linear vibration). The calculations have been carried out for $\nu=0.3$, $\kappa=5 / 6$ and $H=0$, respectively.

The backbone curves for various slenderness ratios for two modes of vibration of a simply-supported Timoshenko beam are shown in Fig. 1.

For the first mode $(k=1), \alpha$ is the amplitude in the middle of the beam $(\xi=1 / 2)$, for $k=2$, $\xi=1 / 4$ ( $L$ is the beam length). One can see that the frequency ratio for the simply-supported Timoshenko beam depends on the kind of mode. 
Table 1. Non-linear frequency ratio $\omega / \omega_{1}$ for simply-supported continuous beams

\begin{tabular}{|c|c|c|c|c|c|c|c|c|}
\hline \multirow{3}{*}{$\alpha / \rho$} & \multicolumn{3}{|c|}{ Bernoulli-Euler beam } & \multicolumn{3}{c|}{ Timoshenko beam } \\
\cline { 2 - 9 } & \multicolumn{2}{|c|}{$\lambda=30$} & \multicolumn{2}{|c|}{$\lambda=10$} & \multicolumn{2}{c|}{$\lambda=20$} & \multicolumn{2}{c|}{$\lambda=30$} \\
\cline { 2 - 9 } & $I_{\rho} \neq 0$ & $I_{\rho}=0$ & $I_{\rho} \neq 0$ & $I_{\rho}=0$ & $I_{\rho} \neq 0$ & $I_{\rho}=0$ & $I_{\rho} \neq 0$ & $I_{\rho}=0$ \\
\hline \hline 1.0 & 1.0897 & 1.0897 & 1.1158 & 1.1159 & 1.0963 & 1.0963 & 1.0927 & 1.0927 \\
\hline 2.0 & 1.3229 & 1.3229 & 1.4068 & 1.4075 & 1.3445 & 1.3445 & 1.3325 & 1.3325 \\
\hline 3.0 & 1.6394 & 1.6394 & 1.7889 & 1.7908 & 1.6785 & 1.6785 & 1.6559 & 1.6559 \\
\hline 4.0 & 2.0000 & 2.0000 & 2.2146 & 2.2190 & 2.0568 & 2.0569 & 2.0255 & 2.0255 \\
\hline$\Omega_{1}$ & 9.8159 & 9.8696 & 8.3874 & 8.6299 & 9.4106 & 9.5103 & 9.6556 & 9.7050 \\
\hline
\end{tabular}

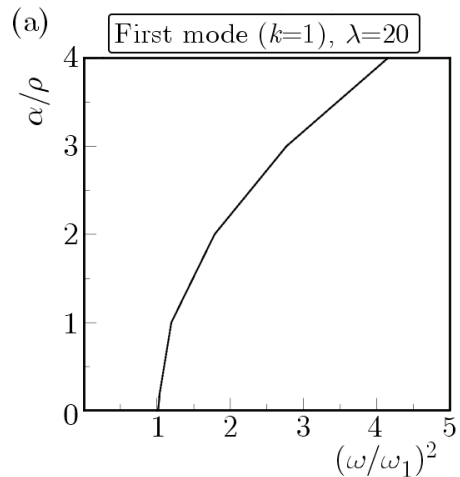

(c)

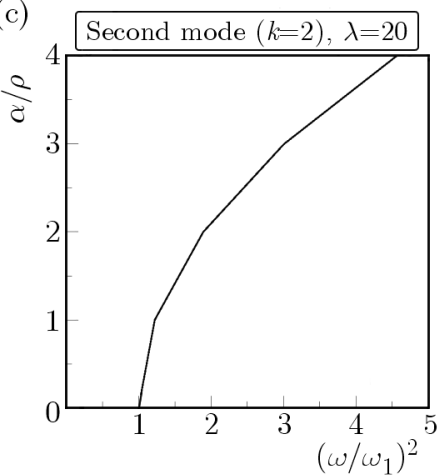

(b) First mode $(k=1), \lambda=100$

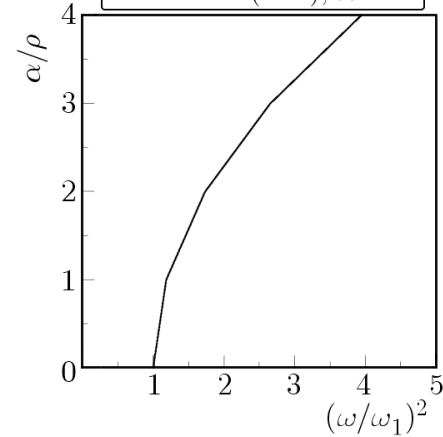

(d)

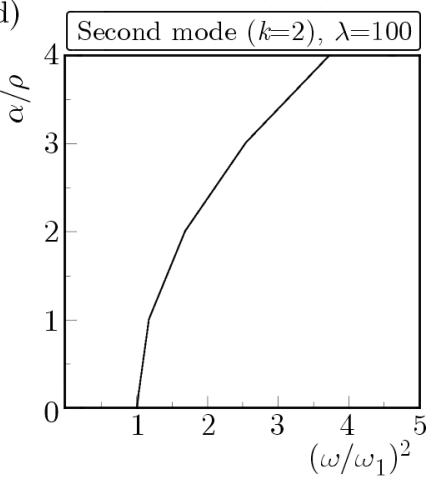

Fig. 1. The backbone curves for various slenderness ratios for two modes of vibration of a simply-supported Timoshenko beam

\section{Finite Element Method formulation}

Let us assume the element shape functions to be linear and independent

$$
\begin{aligned}
& u(x, t)=u_{1}(t)+\left[u_{2}(t)-u_{1}(t)\right] \frac{x}{a} \\
& w(x, t)=w_{1}(t)+\left[w_{2}(t)-w_{1}(t)\right] \frac{x}{a} \\
& \theta(x, t)=\theta_{1}(t)+\left[\theta_{2}(t)-\theta_{1}(t)\right] \frac{x}{a}
\end{aligned}
$$

The beam element convention is presented in Fig. 2 .

Substituting (3.1) into (2.1), we obtain the element stiffness matrix in which the non-linear part corresponding to the nodal quantities $\left\{\begin{array}{lllllll}u_{1} & u_{2} & w_{1} & \theta_{1} & w_{2} & \theta_{2}\end{array}\right\}^{\mathrm{T}}$ can be written in the following form 

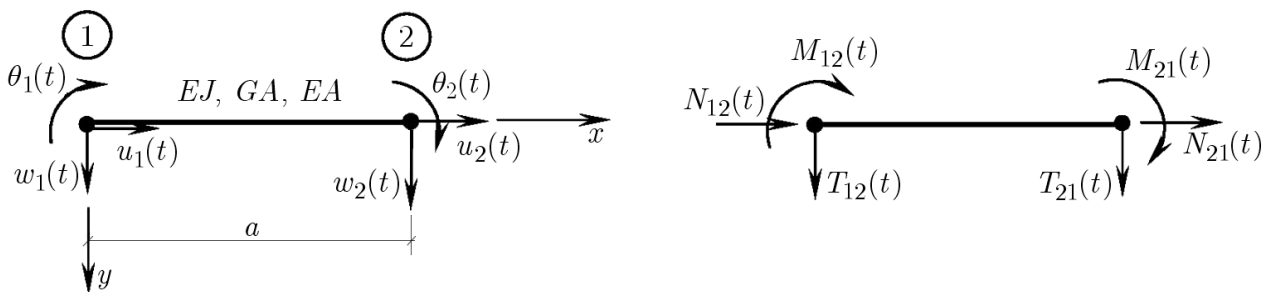

Fig. 2. Timoshenko beam convention

$$
\mathbf{K}_{N}^{*}=\frac{E A}{a}\left[\begin{array}{cccccc}
1 & -1 & \left(w_{2}-w_{1}\right) /(2 a) & 0 & -\left(w_{2}-w_{1}\right) /(2 a) & 0 \\
-1 & 1 & -\left(w_{2}-w_{1}\right) /(2 a) & 0 & \left(w_{2}-w_{1}\right) / a & 0 \\
\left(w_{2}-w_{1}\right) / a & -\left(w_{2}-w_{1}\right) / a & \left(w_{2}-w_{1}\right)^{2} /\left(2 a^{2}\right) & 0 & -\left(w_{2}-w_{1}\right)^{2} /\left(2 a^{2}\right) & 0 \\
0 & 0 & 0 & 0 & 0 & 0 \\
-\left(w_{2}-w_{1}\right) / a & \left(w_{2}-w_{1}\right) / a & -\left(w_{2}-w_{1}\right)^{2} /\left(2 a^{2}\right) & 0 & \left(w_{2}-w_{1}\right)^{2} /\left(2 a^{2}\right) & 0 \\
0 & 0 & 0 & 0 & 0 & 0
\end{array}\right]
$$

Neglecting the horizontal forces in the calculations yields for each element the following relationship

$$
C=\frac{E A}{a}\left[u_{1}-u_{2}+\frac{w_{1}\left(w_{2}-w_{1}\right)}{2 a}-\frac{w_{2}\left(w_{2}-w_{1}\right)}{2 a}\right]=\text { const }
$$

Assuming that the considered Timoshenko beam with immovable supports is divided into elements with equally spaced nodes and summing up both sides of equation (3.2) in the range from 0 to $R$ ( $R$ is the number of beam elements), we have

$$
C=\frac{E A}{2 R a} \sum_{r=0}^{R-1}\left(w_{r+1}-w_{r}\right)^{2}
$$

In order to avoid the shear locking which occurs in linear elements and leads to erroneous results, we improve the elements by introducing a scaling factor $d /(d+1)$ into the flexural and shear matrices (Rakowski, 1990). Replacing $E$ and $G$ by $E d /(d+1)$ and $G d /(d+1)$, respectively, and eliminating the unknowns $u_{i}$, the element stiffness matrix can be expressed as follows

$$
\mathbf{K}=\frac{E J}{a^{3}} \frac{1}{d+1}\left[d \mathbf{K}_{F}+\mathbf{K}_{S}+(d+1)\left(\mathbf{K}_{G}+\mathbf{K}_{N}\right)\right]
$$

where $d=\left[24(1+\nu) \rho^{2}\right] /\left(\kappa a^{4}\right), \mathbf{K}_{F}, \mathbf{K}_{S}, \mathbf{K}_{G}$ and $\mathbf{K}_{N}$ are the flexural, shear, geometrical and non-linear matrices, respectively. They refer to the nodal quantities $\left\{\begin{array}{llll}w_{1} & a \theta_{1} & w_{2} & a \theta_{2}\end{array}\right\}^{\mathrm{T}}$ and have the form

$$
\begin{array}{rlr}
\mathbf{K}_{F}=\left[\begin{array}{cccc}
0 & 0 & 0 & 0 \\
0 & 1 & 0 & -1 \\
0 & 0 & 0 & 0 \\
0 & -1 & 0 & 1
\end{array}\right] & \mathbf{K}_{S}=\left[\begin{array}{cccc}
12 & 6 & -12 & 6 \\
6 & 4 & -6 & 2 \\
-12 & -6 & 12 & -6 \\
6 & 2 & -6 & 4
\end{array}\right] \\
\mathbf{K}_{G}=\frac{a^{2}}{E J}\left[\begin{array}{cccc}
H & 0 & -H & 0 \\
0 & 0 & 0 & 0 \\
-H & 0 & H & 0 \\
0 & 0 & 0 & 0
\end{array}\right] & \mathbf{K}_{N}=\frac{a^{2}}{E J}\left[\begin{array}{cccc}
C & 0 & -C & 0 \\
0 & 0 & 0 & 0 \\
-C & 0 & C & 0 \\
0 & 0 & 0 & 0
\end{array}\right]
\end{array}
$$


We assume the nodal displacements to be harmonic

$$
w_{r}=\alpha W_{r} \cos \omega t \quad \theta_{r}=\frac{\alpha}{a} \phi_{r} \cos \omega t
$$

where $\alpha$ is the amplitude of an arbitrarly chosen node $i, W_{r}$ and $\phi_{r}$ are dimensionless functions of the discrete variable $r$ ( $r$ is the index number of the node), $r \in\langle 0, R\rangle$. By using the shape functions given in (3.1), the consistent matrix is obtained as

$$
\mathbf{M}_{C}=\frac{a m}{6}\left[\begin{array}{cccc}
2 & 0 & 1 & 0 \\
0 & 2 \rho^{2} / a^{2} & 0 & \rho^{2} / a^{2} \\
1 & 0 & 2 & 0 \\
0 & \rho^{2} / a^{2} & 0 & 2 \rho^{2} / a^{2}
\end{array}\right]
$$

If the beam, divided into a regular mesh, consists of identical elements, the dynamic equilibrium conditions can be derived according to the following way. We substitute (3.5) into (3.4) and take into account element mass matrix (3.7). Having assembled the two adjacent beam elements $(r-1, r)$ and $(r, r+1)$, we can write the equilibrium of moments and forces for an arbitrary node $r$ as

$$
\begin{aligned}
& 6\left(W_{r-1}-W_{r}\right)+2\left(\phi_{r-1}+2 \phi_{r}\right)+6\left(W_{r}-W_{r+1}\right)+2\left(2 \phi_{r}+\phi_{r+1}\right)-d\left(\phi_{r-1}+\phi_{r}\right) \\
& \quad+d\left(\phi_{r}-\phi_{r+1}\right)-4 \Lambda^{2}(d+1) e^{2}\left(\phi_{r-1}+4 \phi_{r}+\phi_{r+1}\right)=0 \\
& -[12+6(d+1)]\left(W_{r-1}-W_{r}\right)+[12+6(d+1) D]\left(W_{r}-W_{r+1}\right)-6\left(\phi_{r-1}-\phi_{r}\right) \\
& \quad+6\left(\phi_{r}-\phi_{r+1}\right)-4 \Lambda^{2}(d+1)\left(W_{r-1}+4 W_{r}+W_{r+1}\right)=0
\end{aligned}
$$

Introducing the shifting operator $\mathrm{E}_{r}^{n}$ and central difference operator $\Delta_{r}^{n}$ into equations (3.8), we obtain two difference equations with unknown nodal variables $\phi_{r}$ and $W_{r}$

$$
\begin{aligned}
& {\left[\left(\Delta^{2}+6\right)-\frac{d}{2} \Delta^{2}\right] \phi_{r}-3\left(\mathrm{E}-\mathrm{E}^{-1}\right) W_{r}-2 \Lambda^{2}(d+1) e^{2}\left(\Delta^{2}+6\right) \phi_{r}=0} \\
& \left(\mathrm{E}-\mathrm{E}^{-1}\right) \phi_{\mathrm{r}}-2[2+\mathrm{D}(\mathrm{d}+1)] \Delta^{2} \mathrm{~W}_{\mathrm{r}}-\frac{2}{3} \Lambda^{2}(\mathrm{~d}+1)\left(\Delta^{2}+6\right) \mathrm{W}_{\mathrm{r}}=0
\end{aligned}
$$

where

$$
\begin{array}{lll}
\Delta^{2}=\Delta_{r}^{2}=\mathrm{E}+\mathrm{E}^{-1}-2 & \mathrm{E}^{n}=\mathrm{E}_{r}^{n} & \Lambda=\frac{\Omega^{2}}{24 R^{4}} \\
\Omega^{2}=\frac{m \omega^{2} L^{4}}{E J} & D=\frac{a^{2}(H+C)}{6 E J} & e=\frac{R}{\lambda}
\end{array}
$$

$L$ is length of the beam $(L=a R), \lambda$ is the slenderness ratio $(\lambda=L / \rho$.

Equating to zero the weighed residual of equation (3.9) for one time period $(0-2 \pi)$ with the weighting function cos $\omega t$ (Marur and Prathap, 2005)

$$
\int_{0}^{2 \pi} \varepsilon_{i}(r, t) \cos \omega t d(\omega t)=0 \quad i=1,2
$$

$\left(\varepsilon_{1}, \varepsilon_{2}\right.$ are the left-hand sides of the first and second equation of (3.9) multiplied by $\left.\cos \omega t\right)$ one obtains the equilibrium conditions given in the form of equations (3.9) where now

$$
D=\frac{a^{2}}{6 E J}\left(H+\frac{3}{4} C\right) \quad C=\frac{E A R \delta^{2}}{2 \lambda^{2}} \sum_{r=0}^{R-1}\left(W_{r+1}-W_{r}\right)^{2}
$$


After elimination of $\phi_{r}$ from coupled equation (3.9), we can rewrite them in form of a single fourth-order homogenous difference equation

$$
B_{1} \Delta^{4} W_{r}+B_{2} \Delta^{2} W_{r}+B_{3} W_{r}=0
$$

where

$$
\begin{aligned}
& B_{1}=1-D\left(1-\frac{d}{2}\right)+\frac{\Lambda^{2}}{3}\left(d-2+12 e^{2}\right)+2 \Lambda^{2} D e^{2}(d+1)+\frac{4}{3} \Lambda^{4} e^{2}(d+1) \\
& B_{2}=-6 D+2 \Lambda^{2}\left(d-4+12 e^{2}\right)+12 \Lambda^{2} D e^{2}(d+1)+16 \Lambda^{4} e(d+1) \\
& B_{3}=-24 \Lambda^{2}+48 \Lambda^{4} e^{2}(d+1)
\end{aligned}
$$

The analytical solution to equation (3.12) is

$$
W_{r}=C_{1} \sinh \mu_{1} r+C_{2} \cosh \mu_{1} r+C_{3} \sinh \mu_{2} r+C_{4} \cosh \mu_{2} r
$$

where $\mu_{1}, \mu_{2}$ can be real, imaginary or complex, and they must fulfill the characteristic quadratic equation

$$
B_{1} \cosh ^{2} \mu+\left(-2 B_{1}+\frac{B_{2}}{2}\right) \cosh \mu+B_{1}-B_{2}+\frac{B_{4}}{4}=0
$$

The function $\phi_{r}$ can be derived from the second equation of (3.9) in the form

$$
\phi_{r}=D_{2} \sinh \mu_{1} r+D_{1} \cosh \mu_{1} r+D_{4} \sinh \mu_{2} r+D_{3} \cosh \mu_{2} r
$$

where

$$
\begin{aligned}
& D_{1}=\beta_{1} C_{1} \quad D_{2}=\beta_{1} C_{2} \quad D_{3}=\beta_{2} C_{3} \quad D_{4}=\beta_{2} C_{4} \\
& \beta_{1}=\frac{2}{\sinh \mu_{1}}\left\{\left(\cosh \mu_{1}-1\right)\left[1+\frac{D}{2}(d+1)\right]+\frac{\Lambda^{2}}{3}\left(\cosh \mu_{1}+2\right)(d+1)\right\} \\
& \beta_{2}=\frac{2}{\sinh \mu_{2}}\left\{\left(\cosh \mu_{2}-1\right)\left[1+\frac{D}{2}(d+1)\right]+\frac{\Lambda^{2}}{3}\left(\cosh \mu_{2}+2\right)(d+1)\right\}
\end{aligned}
$$

If a lumped mass model of the Timoshenko beam is considered, the element mass matrix has the following form

$$
\mathbf{M}_{1}=\frac{a m}{2}\left[\begin{array}{cccc}
1 & 0 & 1 & 0 \\
0 & \rho^{2} /+a^{2} & 0 & \rho^{2} / a^{2} \\
1 & 0 & 1 & 0 \\
0 & \rho^{2} / a^{2} & 0 & \rho^{2} / a^{2} a^{2}
\end{array}\right]
$$

The difference equilibrium equations corresponding to element mass matrix $\mathbf{M}_{1}$ (3.16) are

$$
\begin{aligned}
& {\left[\left(\Delta^{2}+6\right)-\frac{d}{2} \Delta^{2}\right] \phi_{r}-3\left(\mathrm{E}-\mathrm{E}^{-1}\right) \mathrm{W}_{\mathrm{r}}-12 \Lambda^{2} \mathrm{e}^{2}(\mathrm{~d}+1) \phi_{\mathrm{r}}=0} \\
& \left(\mathrm{E}-\mathrm{E}^{-1}\right) \phi_{r}-2[2+D(d+1)] \Delta^{2} W_{r}-4 \Delta^{2}(d+1) W_{r}=0
\end{aligned}
$$

or expressed in form of an equation with one unknown $W_{r}$

$$
B_{1} \Delta^{4} W_{r}+B_{2} \Delta^{2} W_{r}+B_{3} W_{r}=0
$$

where

$$
\begin{aligned}
& B_{1}=1-D\left(1-\frac{d}{2}\right) \quad B_{2}=-6 D+2 \Lambda^{2}\left[d-2+12 e^{2}+6 D e^{2}(d+1)\right] \\
& B_{3}=-24 \Lambda^{2}+48 \Lambda^{4} e^{2}(d+1)
\end{aligned}
$$


The solution to this equation and the expression of the characteristic equation are identical to those given in (3.13) and (3.14), respectively. But in this case, the quantities $\beta_{i}$ present in the relationship between $D_{i}$ and $C_{i}$ (see (3.15)), derived from (3.17), are defined as

$$
\left.\beta_{1}=\frac{2}{\sinh \mu_{i}}\left\{\cosh \mu_{i}-1\right)\left[1+\frac{D}{2}(d+1)\right]+\Lambda^{2}(d+1)\right\} \quad i=1,2
$$

Since the non-linear frequency parameter $\Lambda$ and the non-linear vibration modes $W_{r}$ and $\phi_{r}$ depend on the amplitude $\alpha$, the eigenvalue problem of non-linear equation (3.12) is solved iteratively. The iteration starts by assuming the magnitude of the constant $D$ (it is convenient in the first step of iteration to use the value calculated for the linear case). From the frequency equations adequate to the considered boundary conditions, one finds $\mu_{1}$ and $\mu_{2}$. Then the vibration mode $W_{r}$ is determined and the resulting parameter $\bar{D}$ is compared with the given $D$. The iterative computation is continued until the difference $|\bar{D}-D|$ is less than the adopted tolerance. The boundary conditions are considered below.

\subsection{Simply-supported beam}

For a simply supported beam with the boundary nodes 0 and $R$, the boundary conditions $W_{0}=W_{R}=0, M_{0}=M_{R}=0$ lead to the following mode of vibrations which is independent of the vibration amplitude

$$
W_{r}=C_{1} \sin (k \pi r)
$$

and $k$ being the number of mode. The frequency parameter $\Lambda$ can be determined as the root of the quadratic equation

$$
a_{k} \Lambda^{2}+b_{k} \Lambda+c_{k}=0
$$

where

$$
\begin{aligned}
a_{k} & =4 e^{2}(d+1)\left\{\frac{1}{3}\left[\cos \left(\frac{k \pi}{R}\right)-1\right]^{2}+2\left[\cos \left(\frac{k \pi}{R}\right)-1\right]+3\right\} \\
b_{k} & =\left[\cos \left(\frac{k \pi}{R}\right)-1\right]^{2}\left[-\frac{2}{3}+\frac{d}{3}+4 e^{2}+2 e^{2}(d+1) D\right] \\
& +\left[\cos \left(\frac{k \pi}{R}\right)-1\right]\left[-4+d+12 e^{2}+6 e^{2}(d+1) D\right]-6 \\
c_{k} & =\left[\cos \left(\frac{k \pi}{R}\right)-1\right]\left\{\left[\cos \left(\frac{k \pi}{R}\right)-1\right]\left[1-D\left(1-\frac{d}{2}\right)\right]-3 D\right\}
\end{aligned}
$$

When the lumped mass model is considered, the above coefficients must be replaced by

$$
\begin{aligned}
a_{k} & =4 e^{2}(d+1) \\
b_{k} & =\left[\cos \left(\frac{k \pi}{R}\right)-1\right]^{2}\left[-2+d+12 e^{2}+6 e^{2}(d+1) D\right]-6 \\
c_{k} & =\left[\cos \left(\frac{k \pi}{R}\right)-1\right]\left\{\left[\cos \left(\frac{k \pi}{R}\right)-1\right]\left[1-D\left(1-\frac{d}{2}\right)\right]-3 D\right\}
\end{aligned}
$$

\subsection{Clamped-clamped beam}

The boundary conditions $W_{0}=W_{R}=0, \phi_{0}=\phi_{R}=0$ request the following equations to be fulfilled

$$
\begin{aligned}
& C_{1}\left[\beta_{2} \sinh \left(\mu_{1} R\right)-\beta_{1} \sinh \left(\mu_{2} R\right)\right]+C_{2} \beta_{2}\left[\cosh \left(\mu_{1} R\right)-\cosh \left(\mu_{2} R\right)\right]=0 \\
& C_{1} \beta_{1}\left[\cosh \left(\mu_{1} R\right)-\cosh \left(\mu_{2} R\right)\right]+C_{2}\left[\beta_{1} \sinh \left(\mu_{1} R\right)-\beta_{2} \sinh \left(\mu_{2} R\right)\right]=0
\end{aligned}
$$


Equating to zero the determinant of the set of equations (3.20) yields the characteristic equation

$$
2 \beta_{1} \beta_{2}\left[\cosh \left(\mu_{1} R\right) \cosh \left(\mu_{2} R\right)-1\right]-\left(\beta_{1}^{2}+\beta_{2}^{2}\right) \sinh \left(\mu_{1} R\right) \sinh \left(\mu_{1} R\right)=0
$$

The modes of vibrations are

$$
W_{r}=C_{1}\left\{\sinh \left(\mu_{1} r\right)-\frac{\beta_{1}}{\beta_{2}} \sinh \left(\mu_{2} r\right)-\beta\left[\cosh \left(\mu_{1} r\right)-\cosh \left(\mu_{2} r\right)\right]\right\}
$$

where

$$
\beta=\frac{\beta_{1}\left[\cosh \left(\mu_{1} R\right)-\cosh \left(\mu_{2} R\right)\right]}{\beta_{1} \sinh \left(\mu_{1} R\right)-\beta_{2} \sinh \left(\mu_{2} R\right)}
$$

\subsection{Hinged-clamped beam}

In this case, the boundary conditions are given by $W_{0}=W_{R}=0, M_{0}=0, \phi_{R}=0$, which refer to the homogenous equations

$$
\begin{aligned}
& C_{1} \sinh \left(\mu_{1} R\right)+C_{3} \sinh \left(\mu_{2} R\right)=0 \\
& C_{1} \beta_{1} \cosh \left(\mu_{1} R\right)+C_{3} \beta_{2} \cosh \left(\mu_{2} R\right)=0
\end{aligned}
$$

and give the characteristic equation

$$
\beta_{2} \tanh \left(\mu_{1} R\right)-\beta_{1} \tanh \left(\mu_{2} R\right)=0
$$

The modes of vibrations can be expressed as

$$
W_{r}=C_{1}\left[\sinh \left(\mu_{1} r\right)-\frac{\sinh \left(\mu_{1} R\right)}{\sinh \left(\mu_{2} R\right)} \sinh \left(\mu_{2} r\right)\right]
$$

Let us now analyse the reduced-integrated linear elements. When the one-point quadrature is applied to the shear term of strain energy (2.1), the element stiffness matrix becomes

$$
\mathbf{K}=\frac{E J}{a^{3}} \frac{1}{d}\left[(d-1) \mathbf{K}_{F}+\mathbf{K}_{S}+d\left(\mathbf{K}_{G}+\mathbf{K}_{N}\right)\right]
$$

where $\mathbf{K}_{F}, \mathbf{K}_{S}, \mathbf{K}_{G}$ and $\mathbf{K}_{N}$ are defined as in (3.4). The difference between the above matrix and that given in (3.4) is that in (3.26) the expression $(d-1)$ is used instead of $d$.

\section{Numerical examples}

The non-linear frequency ratios are determined for beams with various boundary conditions and for a wide spectrum of the thickness ratio. The consistent and lumped mass model of beams are considered. The results of computation are compared with the results obtained for linear elements to which the reduced-selective integration technique has been applied. The calculations have been carried out with the values $\nu=0.3, \kappa=5 / 6$ and $H=0$. The following indications are accepted:

- CONT - the beam with continuous mass distribution,

- MOD - the beam divided into modified finite elements proposed by Rakowski (1990),

- RI - the beam divided into linear elements with reduced integration correction.

Numerical results are obtained for the lumped mass model. Values in the parentheses refer to the consistent mass model. 
Table 2. Comparison of the frequency ratio $\left(\omega / \omega_{1}\right)$ for continuous and discrete $(R=8)$ simply-supported Timoshenko beams

\begin{tabular}{|c|c|c|c|c|c|c|}
\hline \multirow{2}{*}{$\alpha / \rho$} & \multicolumn{7}{|c|}{$\lambda=20$} & \multicolumn{3}{c|}{$\lambda=100$} \\
\cline { 2 - 7 } & CONT & MOD & RI & CONT & MOD & RI \\
\hline \hline \multicolumn{7}{|c|}{ First mode $(k=1)$} \\
\hline$\Omega_{1}$ & 9.411 & 9.528 & 9.584 & 9.850 & 9.977 & 10.042 \\
& & $(9.406)$ & $(9.461)$ & & $(9.849)$ & $(9.914)$ \\
\hline 1.0 & 1.0963 & 1.0941 & 1.0930 & 1.0900 & 1.0878 & 1.0867 \\
\hline 2.0 & 1.3445 & 1.3371 & 1.3336 & 1.3237 & 1.3165 & 1.3129 \\
\hline 3.0 & 1.6785 & 1.6652 & 1.6587 & 1.6409 & 1.6278 & 1.6212 \\
\hline 4.0 & 2.0568 & 2.0375 & 2.0281 & 2.0023 & 1.9832 & 1.9736 \\
\hline \multicolumn{7}{|c|}{ Second mode $(k=2)$} \\
\hline$\Omega_{1}$ & 33.550 & 35.115 & 35.772 & 39.162 & 41.201 & 42.338 \\
& & $(33.357)$ & $(33.981)$ & & $(39.138)$ & $(40.218)$ \\
\hline 1.0 & 1.1158 & 1.1062 & 1.1021 & 1.0908 & 1.0823 & 1.0781 \\
\hline 2.0 & 1.4048 & 1.3763 & 1.3629 & 1.3264 & 1.2983 & 1.2841 \\
\hline 3.0 & 1.7889 & 1.7346 & 1.7108 & 1.6457 & 1.5945 & 1.5685 \\
\hline 4.0 & 2.2146 & 2.1363 & 2.1021 & 2.0092 & 1.9345 & 1.8962 \\
\hline
\end{tabular}

Table 3. Frequency ratio $\left(\omega / \omega_{1}\right)$ of the first mode for the clamped-clamped beam $(R=8)$

\begin{tabular}{|c|c|c|c|c|c|c|}
\hline \multirow{2}{*}{$\alpha / \rho$} & \multicolumn{2}{|c|}{$\lambda=20$} & \multicolumn{2}{c|}{$\lambda=40$} & \multicolumn{2}{c|}{$\lambda=100$} \\
\cline { 2 - 7 } & MOD & RI & MOD & RI & MOD & RI \\
\hline \hline 1.0 & 1.0251 & 1.0242 & 1.0211 & 1.0204 & 1.0202 & 1.0195 \\
& $(1.0251)$ & $(1.0242)$ & $(1.0212)$ & $(1.0294)$ & $(1.0202)$ & $(1.0195)$ \\
\hline 2.0 & 1.0963 & 1.0929 & 1.0817 & 1.0789 & 1.0781 & 1.0756 \\
& $(1.0965)$ & $(1.0931)$ & $(1.0819)$ & $(1.0790)$ & $(1.0783)$ & $(1.0757)$ \\
\hline 3.0 & 1.2045 & 1.1975 & 1.1746 & 1.1689 & 1.1675 & 1.1625 \\
& $(1.2049)$ & $(1.1979)$ & $(1.1750)$ & $(1.1693)$ & $(1.1678)$ & $(1.1628)$ \\
\hline 4.0 & 1.3400 & 1.3286 & 1.2918 & 1.2830 & 1.2807 & 1.2734 \\
& $(1.3407)$ & $(1.3294)$ & $(1.2925)$ & $(1.2836)$ & $(1.2814)$ & $(1.2739)$ \\
\hline 5.0 & 1.4952 & 1.4789 & 1.4265 & 1.4144 & 1.4114 & 1.4021 \\
& $(1.4962)$ & $(1.4800)$ & $(1.4276)$ & $(1.4154)$ & $(1.4123)$ & $(1.4028)$ \\
\hline$\Omega_{1}$ & 19.082 & 19.539 & 21.609 & 22.281 & 22.529 & 23.295 \\
& $(18.801)$ & $(19.247)$ & $(21.282)$ & $(21.937)$ & $(22.185)$ & $(22.932)$ \\
\hline & \multicolumn{7}{|c|}{ CONT } \\
\hline$\Omega_{1}$ & 18.837 & \multicolumn{6}{c|}{21.296} & 22.189 \\
\hline
\end{tabular}

\section{Concluding remarks}

The analytical solution to the non-linear free vibration problem formulated by the finite element method for the Timoshenko beam is presented. The simple modified linear element is used in the solution. This improved element gives a better convergence to the solution for the continuous system than the reduced-integrated one. The good accuracy is achieved for a wide range of the thickness ratio for the consistent and lumped mass models of the beam. The concept of the finite difference method is introduced. The main advantage of the presented idea is that even for a large number of elements it suffices to consider the non-linear eigenvalue problem of only one difference equation which is equivalent to a set of FEM equilibrium conditions. 
Table 4. Frequency ratio $\left(\omega / \omega_{1}\right)$ of the second mode for the clamped-clamped beam $(R=8)$

\begin{tabular}{|c|c|c|c|c|c|c|}
\hline \multirow{2}{*}{$\alpha / \rho$} & \multicolumn{2}{|c|}{$\lambda=20$} & \multicolumn{2}{c|}{$\lambda=40$} & \multicolumn{2}{c|}{$\lambda=100$} \\
\cline { 2 - 7 } & MOD & RI & MOD & RI & MOD & RI \\
\hline \hline 1.0 & 1.0649 & 1.0604 & 1.0452 & 1.0413 & 1.0402 & 1.0367 \\
& $(1.0652)$ & $(1.0608)$ & $(1.0457)$ & $(1.0418)$ & $(1.407)$ & $(1.10371)$ \\
\hline 2.0 & 1.2372 & 1.2216 & 1.1680 & 1.1540 & 1.1502 & 1.1379 \\
& $(1.2383)$ & $(1.2228)$ & $(1.1697)$ & $(1.1557)$ & $(1.1519)$ & $(1.1393)$ \\
\hline 3.0 & 1.4780 & 1.4480 & 1.3431 & 1.3154 & 1.3079 & 1.2845 \\
& $(1.4796)$ & $(1.4499)$ & $(1.3462)$ & $(1.3188)$ & $(1.3114)$ & $(1.2875)$ \\
\hline 4.0 & 1.7591 & 1.7134 & 1.5506 & 1.5068 & 1.4950 & 1.4590 \\
& $(1.7611)$ & $(1.7158)$ & $(1.5552)$ & $(1.5120)$ & $(1.5004)$ & $(1.4641)$ \\
\hline 5.0 & 2.0640 & 2.0024 & 1.7790 & 1.7169 & 1.7003 & 1.6499 \\
& $(2.0662)$ & $(2.0050)$ & $(1.7846)$ & $(1.7236)$ & $(1.7074)$ & $(1.6572)$ \\
\hline$\Omega_{1}$ & 46.229 & 48.146 & 58.332 & 62.197 & 63.945 & 69.172 \\
& $(43.863)$ & $(45.585)$ & $(55.152)$ & $(58.717)$ & $(60.409)$ & $(65.225)$ \\
\hline & \multicolumn{7}{|c|}{ CONT } & \multicolumn{3}{c|}{55.423} \\
\hline$\Omega_{1}$ & 44.330 & \multicolumn{7}{|c|}{60.519} \\
\hline
\end{tabular}

Table 5. Frequency ratio $\left(\omega / \omega_{1}\right)$ of the third mode for the clamped-clamped beam $(R=8)$

\begin{tabular}{|c|c|c|c|c|c|c|}
\hline \multirow{2}{*}{$\alpha / \rho$} & \multicolumn{2}{|c|}{$\lambda=20$} & \multicolumn{2}{|c|}{$\lambda=40$} & \multicolumn{2}{|c|}{$\lambda=100$} \\
\hline & MOD & RI & MOD & $\mathrm{RI}$ & MOD & $\mathrm{RI}$ \\
\hline 1.0 & $\begin{array}{c}1.1095 \\
(1.1117)\end{array}$ & $\begin{array}{c}1.0989 \\
(1.1009)\end{array}$ & $\begin{array}{c}1.0586 \\
(1.0603)\end{array}$ & $\begin{array}{c}1.0473 \\
(1.0489)\end{array}$ & $\begin{array}{c}1.0442 \\
(1.0457)\end{array}$ & $\begin{array}{c}1.0335 \\
(1.0347)\end{array}$ \\
\hline 2.0 & $\begin{array}{c}1.3768 \\
(1.3799)\end{array}$ & $\begin{array}{c}1.3433 \\
(1.3461)\end{array}$ & $\begin{array}{c}1.2124 \\
(1.2126)\end{array}$ & $\begin{array}{c}1.1737 \\
(1.1777)\end{array}$ & $\begin{array}{c}1.1630 \\
(1.1671)\end{array}$ & $\begin{array}{c}1.1254 \\
(1.1290)\end{array}$ \\
\hline 3.0 & $\begin{array}{c}1.7252 \\
(1.7262)\end{array}$ & $\begin{array}{c}1.6652 \\
(1.6657)\end{array}$ & $\begin{array}{c}1.4268 \\
(1.4310)\end{array}$ & $\begin{array}{c}1.3532 \\
(1.3581)\end{array}$ & $\begin{array}{c}1.3329 \\
(1.3384)\end{array}$ & $\begin{array}{c}1.2599 \\
(1.2652)\end{array}$ \\
\hline 4.0 & $\begin{array}{c}2.1172 \\
(2.1133)\end{array}$ & $\begin{array}{c}2.0290 \\
(2.0241)\end{array}$ & $\begin{array}{c}1.6793 \\
(1.6825)\end{array}$ & $\begin{array}{c}1.5673 \\
(1.5717)\end{array}$ & $\begin{array}{c}1.5367 \\
(1.5425)\end{array}$ & $\begin{array}{c}1.4234 \\
(1.4300)\end{array}$ \\
\hline 5.0 & $\begin{array}{c}2.5518 \\
(2.5283)\end{array}$ & $\begin{array}{c}2.4235 \\
(2.4042)\end{array}$ & $\begin{array}{c}1.9562 \\
(1.9579)\end{array}$ & $\begin{array}{c}1.8047 \\
(1.8082)\end{array}$ & $\begin{array}{c}1.7774 \\
(1.7694)\end{array}$ & $\begin{array}{c}1.6234 \\
(1.6152)\end{array}$ \\
\hline$\Omega_{1}$ & $\begin{array}{r}81.697 \\
(72.825) \\
\end{array}$ & $\begin{array}{c}85.847 \\
(76.475) \\
\end{array}$ & $\begin{array}{l}112.487 \\
(99.927) \\
\end{array}$ & $\begin{array}{c}124.642 \\
(110.404)\end{array}$ & $\begin{array}{c}130.900 \\
(116.090)\end{array}$ & $\begin{array}{c}151.802 \\
(133.961)\end{array}$ \\
\hline & \multicolumn{6}{|c|}{ CONT } \\
\hline$\Omega_{1}$ & & & 101 & 659 & 117 & \\
\hline
\end{tabular}

\section{References}

1. Asghari M., Kahrobaiyan M.H., Ahmadian M.T., 2010, A nonlinear Timoshenko beam formulation based on the modified couple stress theory, International Journal of Engineering Science, 48, 1749-1776

2. Bhashyam G.R., Prathap G., 1980, Galerkin finite element method for non-linear beam vibrations, Journal of Sound and Vibration, 72, 191-203

3. Bhashyam G.R., Prathap G., 1981, The second frequency spectrum of Timoshenko beams, Journal of Sound and Vibration, 76, 407-420

4. Dumir P.C., Bhaskar A., 1988, Some erroneous finite element formulations of non-linear vibrations of beams and plates, Journal of Sound and Vibration, 123, 517-527 
5. Evensen D.A., 1968, Non-linear vibrations of beams with various boundary conditions, American Institute of Aeronatics and Astronomics Journal, 6, 370-372

6. Hsu C.S., 1960, On the application of elliptic functions in non-linear forced oscillations, Quarterly of Applied Mathematics, 17, 393-407

7. Kitipornchai S., Ke L.L., Yang J., Xiang Y., 2009, Nonlinear vibration of edge cracked functionally graded Timoshenko beams, Journal of Sound and Vibration, 324, 962-982

8. Lewandowski R., 1987, Application of the Ritz method to the analysis of non-linear free vibration of beams, Journal of Sound and Vibration, 114, 91-101

9. Levinson M., Cooke D.W., 1982, On the two frequency spectra of Timoshenko beams, Journal of Sound and Vibration, 84, 319-326

10. Marur S.R., Prathap G., 2005, Non-linear beam vibration problems and simplifications in finite element models, Computational Mechanics, 35, 352-360

11. Mei C., Decha-Umphai K., 1985, A finite element method for non-linear forced vibrations of beams, Journal of Sound and Vibration, 102, 369-380

12. Rakowski J., 1990, The interpretation of shear locking in beam elements, Computers and Structures, 37, 5, 769-776

13. Rakowski J., 1991a, A critical analysis of quadratic beam finite elements, International Journal for Numerical Methods in Engineering, 31, 949-966

14. Rakowski J., 1991b, A new methodology of evaluation of C0 bending finite elements, Computer Methods in Applied Mechanics and Engineering, 91, 1327-1338

15. Rakowski J., Wielentejczyk P., 1996, Vibrations of infinite periodic beams by finite element method, Zeitschrift für Angewandte Mathemetic und Mechanik, 76, 411-412

16. Rakowski J., Wielentejczyk P., 2002, Dynamic analysis of infinite discrete structures, Foundations of Civil and Environmental Engineering, 3, 91-106

17. Rosenberg R.M., 1966, On non-linear vibrations of systems with many degree of freedom, $A d-$ vanced in Applied Mechanics, 9, 154-242

18. SARma B., VARAdAn T.K., 1983, Lagrange-type formulation for finite element analysis of nonlinear beam vibrations, Journal of Sound and Vibration, 86, 61-70

19. Singh G., Rao G.V., 1998, Nonlinear oscillations of thick asymmetric cross-ply beams, Acta Mechanics, 127, 135-146

20. SzemplińskA-StupnickA W., 1983, Non-linear normal modes and generalized Ritz method in the problems of vibrations of non-linear elastic continuous systems, International Journal of Non-linear Mechanics, 18, 154-242

21. Woinowsky-Krieger S., 1950, The effect of an axial force on the vibration of hinged bars, Journal of Applied Mechanics, 17, 35-36 